\title{
Cinética e Equilíbrio de Adsorção dos Oxiânions Cr (VI), Mo (VI) e Se (VI) pelo Sal de Amônio Quaternário de Quitosana
}

\author{
Viviane A. Spinelli, Mauro C. M. Laranjeira, Valfredo T. Fávere \\ Departamento de Química, UFSC
}

\author{
Irene Y. Kimura \\ Departamento de Ciências, UEM
}

\begin{abstract}
Resumo: O sal quaternário de quitosana foi sintetizado com cloreto de glicidil trimetil amônio. A modificação química foi caracterizada por espectrometria no IV, $\mathrm{RMN} \mathrm{de}{ }^{13} \mathrm{C} \mathrm{e}{ }^{1} \mathrm{H}, \mathrm{e}$ mmol/g de grupos quaternários presentes na matriz polimérica por condutimetria. A remoção de $\mathrm{Cr}$ (VI), Mo (VI) e Se (VI), em meio aquoso, foi investigada em processo de batelada. A adsorção mostrou ser dependente do pH para o Cr (VI) e Se (VI), com um pH ótimo de adsorção, entre 4,0 a 6,0. Para o Mo (VI) a adsorção manteve-se quase constante no intervalo de pH entre 4,0 e 11,5. O modelo de isoterma de Langmuir descreveu melhor os dados de equilíbrio na faixa de concentração investigada. No presente estudo, um grama do sal quaternário de quitosana reticulado com glutaraldeído adsorveu $68,3 \mathrm{mg}$ de $\mathrm{Cr}, 63,4 \mathrm{mg}$ de Mo e 90,0 mg de Se. A velocidade de adsorção, no processo, segue a equação cinética de pseudo segunda-ordem, sendo que o equilíbrio para os três íons foi alcançado próximo aos 200 minutos. A análise dispersiva de raios-X para o $\mathrm{Cr}$ (VI) mostrou que o principal mecanismo de adsorção é a troca iônica entre os íons $\mathrm{Cl}^{-}$da superfície do polímero pelos oxiânions. $\mathrm{O}$ trocador aniônico apresentou a seguinte ordem de seletividade: $\mathrm{Cr}(\mathrm{VI})>\mathrm{Mo}(\mathrm{VI})>\mathrm{Se}(\mathrm{VI})$.
\end{abstract}

Palavras-chave: Adsorção, cromo (VI), molibdênio (VI), selênio (VI), sal quaternário de quitosana.

\section{Kinetics and Equilibrium of Adsorption of Oxyanions $\mathrm{Cr}$ (VI), Mo (VI) and Se (VI) by Quaternary Ammonium Chitosan Salt}

\begin{abstract}
Quaternary chitosan salt was synthesized in the presence of glycidyl trimetyl ammonium chloride. The polymer was characterized by spectroscopic techniques: infrared, ${ }^{13} \mathrm{C}$ and ${ }^{1} \mathrm{H}$ NMR, while the amount of quaternary ammonium groups was obtained by condutimetry. The removal of $\mathrm{Cr}$ (VI), Mo (VI) and Se (VI) from aqueous solutions was carried out in batch adsorption processes. The process seemed to be $\mathrm{pH}$ dependent for $\mathrm{Cr}$ (VI) and Se (VI) with an optimum $\mathrm{pH}$ ranging from 4.0 to 6.0; while for Mo (VI) the adsorption remained almost constant within the range between 4.0 and 11.5. The Langmuir isotherm model provided the best fit of the equilibrium data over the whole concentration investigated. In the experiment one gram of cross-linked quaternary chitosan salt adsorbed $68.3 \mathrm{mg}$ of chromium, $63.4 \mathrm{mg}$ of molybdenum and $90.0 \mathrm{mg}$ of selenium. The adsorption process followed a pseudo second-order kinetic rate equation and the equilibrium regarding the three ions was reached after 200 minutes. The studies from X-ray dispersive energy showed that the main adsorption mechanism is ionic exchange among $\mathrm{Cl}^{-}$groups on the polymer surface by oxyanions from solution and the anionic exchanger showed the following selectivity order: $\mathrm{Cr}(\mathrm{VI})>\mathrm{Mo}(\mathrm{VI})>\mathrm{Se}(\mathrm{VI})$.
\end{abstract}

Keywords: Adsorption, chromium (VI), molybdenum (VI), selenium (VI), quaternary chitosan salt.

\section{Introdução}

A preocupação e o interesse com as questões ambientais vêm aumentando com o passar das décadas. Cientistas do mundo inteiro vêm desenvolvendo pesquisas que visam avaliar e diminuir os impactos da poluição na biosfera, além de desenvolver tecnologias limpas, que geram menos resíduos. Dentre os vários poluentes, os metais têm recebido atenção especial, uma vez que alguns são extremamente tóxicos, para uma grande variedade de organismos, mesmo em concentrações extremamente baixas. As atividades industriais, agrí- colas e a disposição de rejeitos domésticos contribuem para a liberação de metais no meio ambiente. As indústrias de mineração, as termoelétricas, as de galvanoplastia e os curtumes são as indústrias que mais produzem efluentes com metais tóxicos originários de seus processos ${ }^{[1-3]}$.

$\mathrm{O}$ tratamento clássico de efluentes contendo metais envolve processos físico-químicos de precipitação, troca iônica e eletroquímica. O método mais utilizado atualmente é a precipitação química seguido de sedimentação e filtração. Contudo, essas técnicas tradicionais são inadequadas para a descontaminação de grandes volumes de efluentes

Autor para correspondência: Valfredo T. de Fávere, Departamento de Química, UFSC, Campus Universitário Trindade, CEP: 88040-900, Florianópolis, SC. E-mail: favere@qmc.ufsc.br 
contendo metais em baixas concentrações, devido à baixa eficiência operacional e aos elevados custos deste proces$\mathrm{so}^{[4]}$. Diante disso, métodos alternativos vêm sendo investigados como, por exemplo, eletrodiálise, osmose reversa, ultrafiltração e a adsorção com biosorventes. A grande vantagem da adsorção sobre as outras é a baixa geração de resíduos, fácil recuperação dos metais e a possibilidade de reutilização do adsorvente ${ }^{[5]}$.

Dentre os materiais naturais, a quitosana destaca-se como um efetivo adsorvente de metais de transição $0^{[6-9]}$, a qual é obtida em escala industrial pela desacetilação alcalina da quitina, um dos biopolímeros mais abundantes na natureza. A versatilidade da quitosana é atribuída aos grupos amino livres que ficam expostos após a reação de desacetilação da quitina. Várias modificações químicas têm sido realizadas na quitosana para facilitar a transferência de massa, expor os sítios ativos de ligação, aumentar a capacidade e seletividade de adsorção e reduzir sua solubilidade em água ${ }^{[6-12]}$.

A presente investigação tem como objetivo, modificar quimicamente a superfície da quitosana com cloreto de trimetil glicidil amônio ${ }^{[12]}$, para obter um sal de amônio quaternário de quitosana, o qual terá sítios catiônicos; e avaliar as propriedades de equilíbrio de adsorção dos oxiânions cromo (VI), molibdênio (VI) e selênio (VI).

\section{Experimental}

\section{Materiais}

A quitosana com grau de desacetilação de $90 \%$ (condutimetria) e massa molar média de $122,7 \times 10^{3} \mathrm{~g} \mathrm{~mol}^{-1}$ (viscosimetria) determinadas em nosso laboratório, foi adquirida da empresa Purifarma. O reagente cloreto de trimetil glicidil amônio foi obtido da Fluka Chemica. Todos os reagentes usados foram de grau analítico. A solução padrão de Cr (VI) de $1000 \mathrm{mg} \mathrm{L}^{-1}$ foi preparada a partir do padrão primário dicromato de potássio p.a. (Synth, Brasil) e as soluções de Mo (VI) e Se (VI) foram preparadas usando solução de titrisol $1000 \mathrm{mg} \mathrm{L}^{-1}$ (Merck, Brasil). As soluções de calibração dos metais $\mathrm{Cr}$, Mo e Se foram preparadas a partir de diluições adequadas das soluções padrão de cada metal.

\section{Síntese do sal de amônio quaternário de Quitosana}

O sal de amônio quaternário de quitosana (SAQQ), foi sintetizado pela dispersão em meio aquoso ( $30 \mathrm{~g}$ de quitosana em $300 \mathrm{~mL}$ de água). A esta mistura foram adicionados $60,6 \mathrm{~g}$ de cloreto de glicidil trimetil amônio e o sistema permaneceu em contato sob agitação, a $60{ }^{\circ} \mathrm{C}$ durante $24 \mathrm{~h}$. O produto da reação foi então precipitado com acetona, filtrado e seco a $60^{\circ} \mathrm{C}^{[12]}$. Após a caracterização, o material foi reticulado com glutaraldeído para inibir sua solubilização em meio aquoso. Treze gramas de SAQQ foram suspensos em $200 \mathrm{~mL}$ de etanol e $10 \mathrm{~mL}$ de glutaraldéido a $25 \%$ foram adicionados à suspensão. O sistema permaneceu sob agitação durante 24 horas, à temperatura ambiente. O material foi separado da solução por filtração, lavado com água destilada, seco a $50^{\circ} \mathrm{C}$ e pulverizado em moinho e tamisado para obtenção de partículas cujos

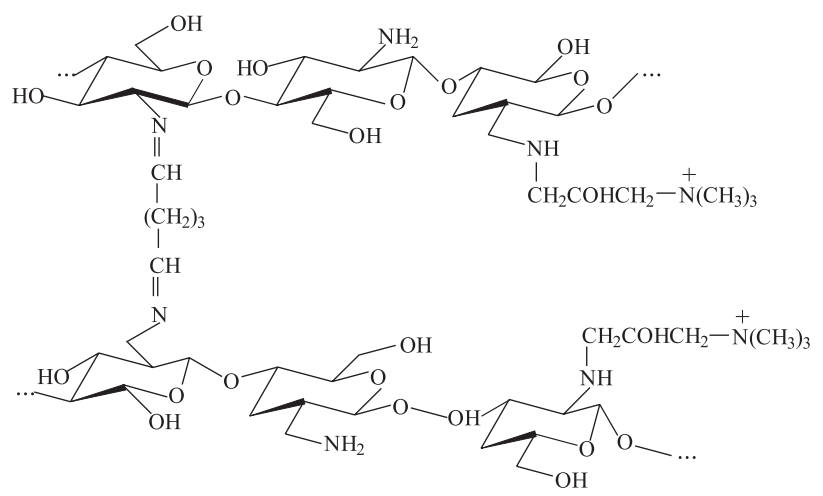

Figura 1. Estrutura do sal de amônio quaternário de quitosana reticulado (SAQQR).

tamanhos vararam entre de 0,150 e $0,212 \mathrm{~mm}$. O sal reticulado (SAQQR) apresentou uma coloração marron-avermelhada. A Figura 1 ilustra a estrutura do polímero sintetizado.

\section{Espectroscopia no infravermelho com transformada de Fourier (FTIR)}

Espectros no infravermelho da quitosana e do sal quaternário de quitosana foram obtidos em pastilhas de $\mathrm{KBr}$ empregando um espectrofotômetro Perkin Elmer PC FTIR 16.

\section{Espectrometria de Ressonância Magnética Nuclear (RMN) de ${ }^{13} \mathrm{C}$ e ${ }^{1} \mathrm{H}$}

As análises de RMN foram realizadas com um espectrofotômetro Bruker AC 200 MHZ em 298 K, solubilizando em água deuterada o polímero antes da reticulação.

\section{Titulação condutimétrica}

A titulação condutimétrica foi conduzida com um condutivímetro Metler MC226 e uma bureta automática Schott Gerat T80/20, para a determinação da quantidade de grupos quaternários imobilizados na quitosana. Os contraíons cloreto presentes no sal quaternário de quitosana foram titulados com solução padrão de $\mathrm{AgNO}_{3}$.

\section{Espectrometria de absorção atômica}

Um espectrômetro de absorção atômica em chama (FAAS), da Varian SpectraAA 50, foi utilizado para a determinação de cromo, molibdênio e selênio nos experimentos de adsorção. Como parâmetro operacional do FAAS utilizou-se o valor fornecido pelo fabricante e somente a vazão do gás para cada metal foi otimizada.

\section{Espectrometria de energia dispersiva (EDX)}

A seletividade e o mecanismo de adsorção foram estudados a partir dos espectros de EDX, empregando um espectrômetro de energia dispersiva de raio-X Philips XL30. A seletividade foi investigada a partir de uma solução contendo $100 \mathrm{mg} \mathrm{L}^{-1}$ dos metais em contato com aproximadamente $200 \mathrm{mg}$ de SQQR (triplicata). Após um tempo prédeterminado o material foi filtrado a seco para a obtenção do espectro de EDX. O mecanismo de adsorção foi investigado utilizando soluções de concentrações de Cr (VI) de 25, 50, 
100 e $1000 \mathrm{mg} \mathrm{L}^{-1}$, em contato com amostras de $100 \mathrm{mg}$ do polímero. As amostras foram filtradas e secas e os espectros de EDX foram obtidos.

\section{Dependência do pH na adsorção}

Estes experimentos foram realizados em erlenmeyers fechados de $125 \mathrm{~mL}$. Várias amostras de $100 \mathrm{mg}$ de SQQR foram colocadas numa série de frascos contendo $50 \mathrm{~mL}$ de solução $100 \mathrm{mg} \mathrm{L}^{-1}$ dos metais. $\mathrm{O} \mathrm{pH}$ foi ajustado na faixa de 4,0 e 12,0 com tampão adequado e o sistema foi colocado num banho termostatizado (Shaker Lab-line) com agitação controlada $(200 \mathrm{rpm})$ a $25^{\circ} \mathrm{C}$ durante 300 minutos. O material foi filtrado e a concentração dos metais no filtrado foi determinada por FAAS.

\section{Efeito do tempo de contato}

A cinética de adsorção dos metais pelo novo material adsorvente foi investigada no $\mathrm{pH}$ ótimo de adsorção de cada metal. Amostras de $500 \mathrm{mg}$ de SQQR foram adicionadas em erlenmeyers fechados de $125 \mathrm{~mL}$, contendo $100 \mathrm{mg} \mathrm{L}^{-1}$ dos metais. Os frascos foram mantidos sob agitação (200 rpm) a $25^{\circ} \mathrm{C}$ e em intervalos de tempos, alíquotas de $200 \mu \mathrm{L}$ foram retiradas e diluídas a $25 \mathrm{~mL}$ com água destilada e as concentrações dos íons foram determinadas por FAAS.

\section{Estudo de equilíbrio de adsorção}

Os estudos de equilíbrio de adsorção foram conduzidos num banho termostatizado a $25^{\circ} \mathrm{C}$, em que $100 \mathrm{mg}$ de SAQQR foram colocados em vários frascos fechados contendo de $10 \mathrm{mgL}^{-1} \mathrm{a}$ $100 \mathrm{mgL}^{-1}$ para o $\mathrm{Cr}$ (VI) e Mo (VI) e $10 \mathrm{mg} / \mathrm{L}$ a $500 \mathrm{mg} / \mathrm{L}$ para o Se (VI), tamponadas em $\mathrm{pH}$ adequado. O sistema foi mantido sob agitação de $200 \mathrm{rpm}$ durante um tempo pré-determinado até atingir o equilíbrio de adsorção. Alíquotas de $200 \mu \mathrm{L}$ foram retiradas e colocadas em balões volumétricos de $25 \mathrm{~mL}$ e a concentração dos metais foram determinadas por FAAS.

\section{Resultados e Discussão}

\section{Caracterização do sal de amônio quaternário de quitosana}

As análises dos espectros no infravermelho, $\mathrm{RMN}$ de ${ }^{13} \mathrm{C}$ e ${ }^{1} \mathrm{H}$, permitiram identificar o sal de amônio quaternário de quitosana sintetizado. As bandas da quitosana e do SAQQ foram muito próximas, exceto naquela encontrada na região de $1482 \mathrm{~cm}^{-1}$. Esta banda corresponde à deformação angular assimétrica dos grupos metila do nitrogênio quaternário, o qual está ausente na quitosana ${ }^{[13-15]}$. Os espectros de RMN de ${ }^{13} \mathrm{C}$ e ${ }^{1} \mathrm{H}$, também apresentaram diferenças marcantes entre o SAQQ e a quitosana. Os espectros RMN de ${ }^{13} \mathrm{C}$ e ${ }^{1} \mathrm{H}$ apresentaram um sinal em 54,7 ppm e em 3,25 ppm, respectivamente e características das metilas no grupo amônio, ausentes no polímero original ${ }^{[13,14,16,17]}$.

A quantidade dos grupos quaternários foi determinada por titulação condutimétrica dos íons cloreto usando uma solução padrão de $\mathrm{AgNO}_{3}$. O valor encontrado após a reticulação do polímero com glutaraldeído foi de 2,09 mmol de grupos quaternários por grama de polímero.

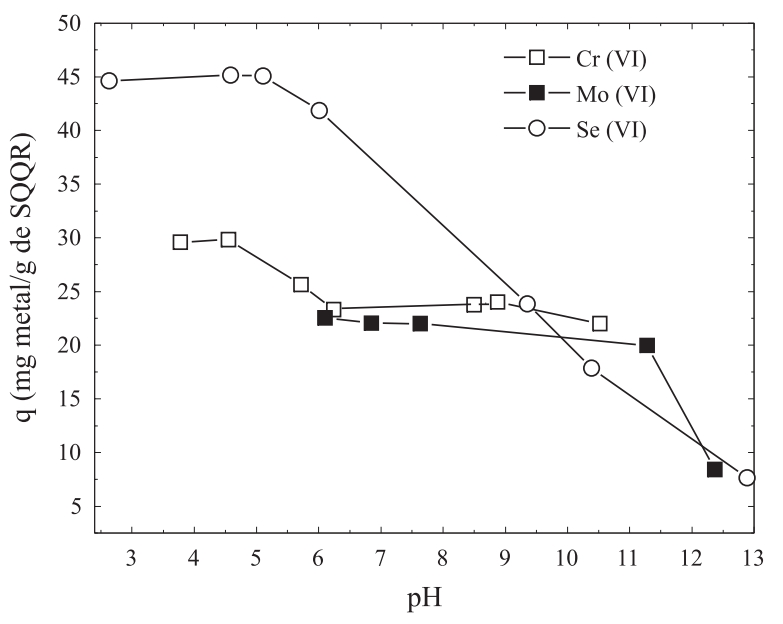

Figura 2. Efeito do pH na adsorção dos oxiânios Cr (VI), Mo (VI) e Se (VI) pelo SAQQR em função da quantidade adsorvida.

$\mathrm{C}_{\mathrm{o}}=100 \mathrm{mg} \mathrm{L}^{-1} ;$ Volume $=50 \mathrm{~mL} ; \mathrm{m}_{\mathrm{SQQR}}=100 \mathrm{mg}$; agitação $=200 \mathrm{rpm}$; temperatura $=25^{\circ} \mathrm{C}$; tempo $=300 \mathrm{~min}$

\section{Efeito do pH na adsorção}

A Figura 2 ilustra o efeito do $\mathrm{pH}$ na adsorção dos oxiânions pelo novo material adsorvente. Pode-se verificar pelo gráfico que a faixa ótima de adsorção dos íons $\mathrm{Cr}$ (VI) e Se (VI), ocorreu no mesmo intervalo de $\mathrm{pH}$, isto é em $\mathrm{pH}$ menor que 6,0. Em pH acima deste, a adsorção do íon $\mathrm{Cr}$ (VI) permaneceu praticamente constante, enquanto o Se (VI) apresentou um decréscimo praticamente linear até $\mathrm{pH}$ fortemente alcalino. Para o Mo (VI) os experimentos não foram conduzidos em pH menor que 6,0 para evitar a precipitação de $\mathrm{MoO}_{3}{ }^{[16]}$. A capacidade de adsorção permaneceu quase constante em $\mathrm{pH}$ entre 6,0 e 11,5 e diminuiu significativamente em $\mathrm{pH}$ 12,0. A dependência do pH revelou para o $\mathrm{Cr}$ (VI) e Se (VI) um equilíbrio prótico no qual as espécies protonadas $\mathrm{HCrO}_{4}{ }^{-}$ e $\mathrm{HSeO}_{4}{ }^{-}$dos oxiânions influenciam a adsorção, enquanto a resina, por ser um trocador fortemente básico, não sofre a influência do $\mathrm{pH}$ da solução.

\section{Cinética de Adsorção}

A Figura 3 ilustra a variação das concentrações dos metais na fase líquida $\left(\mathrm{C}_{\mathrm{t}} / \mathrm{C}_{\mathrm{o}}\right)$ em função do tempo de contato com o adsorvente. $\mathrm{C}_{\mathrm{t}} \mathrm{e} \mathrm{C}_{\mathrm{o}}$ correspondem à concentração dos metais no tempo t e à concentração inicial, respectivamente. Foi observado um decréscimo muito rápido da concentração do Mo (VI) durante os primeiros 5 minutos. Após um período de 200 minutos a concentração dos metais permaneceu quase constante, sendo o equilíbrio de adsorção alcançado depois de 200 minutos para o Mo (VI) e Se (VI) e de 300 minutos para o $\mathrm{Cr}(\mathrm{VI})$.

Para examinar o mecanismo que controla o processo de adsorção, tais como transferência de massa na solução e reação química, vários modelos cinéticos foram testados para interpretar os dados experimentais. Uma boa correlação dos dados cinéticos revela o mecanismo de adsorção. Assim, o coeficiente angular de cada equação cinética foi escolhido para interpretar o mecanismo de adsorção do metal na fase 


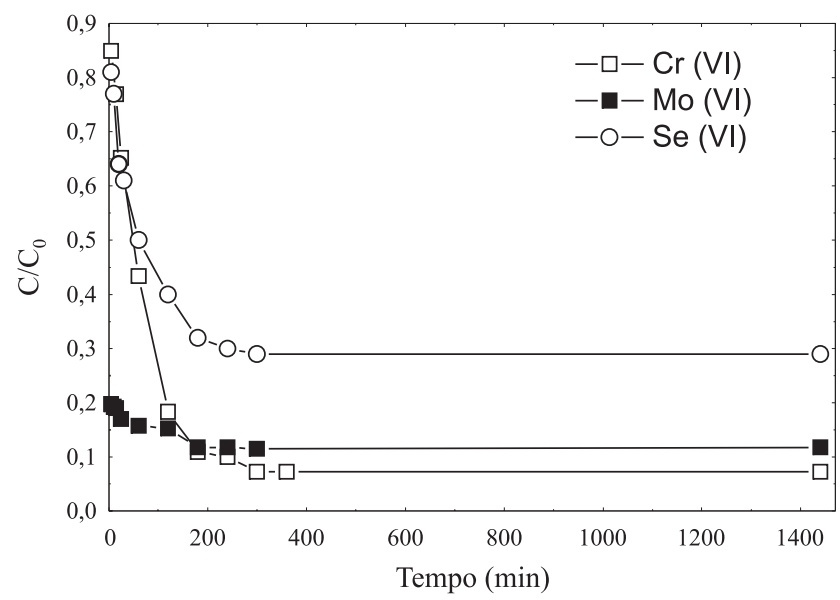

Figura 3. Efeito do tempo de contato na variação da concentração dos oxiânions pela adsorção do SQQR.

$\mathrm{C}_{\mathrm{o}}=100 \mathrm{mg} \mathrm{L}^{-1} ;$ Volume $=10 \mathrm{~mL} ; \mathrm{m}_{\mathrm{SQQR}}=500 \mathrm{mg} ; \mathrm{pH}=4,5(\mathrm{Cr}$ e Se $) \mathrm{e}$ $\mathrm{pH}=8,0(\mathrm{Mo})$; agitação $=200 \mathrm{rpm}$; temperatura $=25^{\circ} \mathrm{C}$

sólida. Os modelos cinéticos de pseudo primeira-ordem, pseudo segunda-ordem e difusão intrapartícula foram testados neste estudo ${ }^{[19-21]}$. A equação de pseudo primeira-ordem é apresentada pela equação 1 .

$$
\mathrm{d} q_{t} / \mathrm{d} t=\mathrm{k}_{1}\left(q_{e}-q_{t}\right)
$$

Em que $\mathrm{k}_{1}$ é a constante de velocidade de pseudo primeiraordem e $\mathrm{q}_{\mathrm{e}}$ é a quantidade adsorvida no equilíbrio. Depois da integração da equação 1 , e aplicando as condições que $\mathrm{q}_{\mathrm{t}}=0$ $a \mathrm{t}=0$ e $\mathrm{q}_{\mathrm{t}}=\mathrm{q}_{\mathrm{t}}$ a $\mathrm{t}=\mathrm{t}$, a equação 1 transforma-se na equação 2 :

$$
\log \left(q_{e}-q_{t}\right)=\log q_{e}-\frac{k_{1}}{2,303} t
$$

A equação de pseudo segunda-ordem pode ser expressa pela equação 3 :

$$
\mathrm{d} q_{\mathrm{t}} / \mathrm{dt}=\mathrm{k}_{2}\left(q_{e}-q_{t}\right)^{2}
$$

Em que $\mathrm{k}_{2}$ é a constante de velocidade de segunda ordem. Fazendo a integração da equação 3 e aplicando as condições iniciais, tem-se :

$$
\frac{1}{\left(q_{e}-q_{t}\right)}=\frac{1}{q_{e}}+k_{2} t
$$

Ou equivalentemente:

$$
\frac{t}{q_{t}}=\frac{1}{k_{2} q_{e}{ }^{2}}+\frac{1}{q_{e}} t
$$

Caso os modelos acima não forneçam um mecanismo definitivo, o modelo de difusão intrapartícula proposto por Weber e Morris pode ser testado ${ }^{[19]}$. O modelo está representado pela equação 6 :

$$
\mathrm{q}_{\mathrm{t}}=\mathrm{ft}^{1 / 2}
$$

A validade destes modelos pode ser checada pelos gráficos lineares de cada equação: $\log \left(\mathrm{q}_{\mathrm{e}}-\mathrm{q}_{\mathrm{t}}\right)$ vs t para o modelo da pseudo primeira-ordem, $\mathrm{t} / \mathrm{q}_{\mathrm{t}}$ vs $\mathrm{t}$ para o modelo de pseudo segunda-ordem e $\mathrm{q}_{\mathrm{t}} \mathrm{vs}^{1 / 2}$ para a difusão intrapartícula, res-
Tabela 1. Parâmetros das regressões lineares determinados empregando os vários modelos de cinética.

\begin{tabular}{llc}
\hline \multicolumn{1}{c}{ Metais } & \multicolumn{1}{c}{ Equação } & R \\
\hline Pr $(\mathrm{VI})$ & $\mathrm{Y}=1,204390-0,00673 \mathrm{X}$ & 0,99042 \\
Mo (VI) & $\mathrm{Y}=0,25993-0,00823 \mathrm{X}$ & 0,98002 \\
Se (VI) & $\mathrm{Y}=1,04472-0,00759 \mathrm{X}$ & 0,99182 \\
\hline & Pseudo segunda-ordem & \\
\hline Cr (VI) & $\mathrm{Y}=2,23952+0,04733 \mathrm{X}$ & 0,99451 \\
Mo (VI) & $\mathrm{Y}=0,11183+0,05776 \mathrm{X}$ & 0,99989 \\
Se (VI) & $\mathrm{Y}=1,26261+0,07175 \mathrm{X}$ & 0,99984 \\
\hline & Difusão intrapartícula & \\
\hline Cr (VI) & $\mathrm{Y}=0,72484+1,19635 \mathrm{X}$ & 0,97921 \\
Mo (VI) & $\mathrm{Y}=15,3295+0,14089 \mathrm{X}$ & 0,97921 \\
Se (VI) & $\mathrm{Y}=3,32537+0,67708 \mathrm{X}$ & 0,96938 \\
\hline
\end{tabular}

pectivamente. A avaliação quantitativa requer que o coeficiente de correlação seja comparado ${ }^{[20,21]}$.

Os parâmetros das regressões lineares determinados empregando os vários modelos e as curvas lineares obtidos são apresentadas na Tabela 1 .

Os estudos cinéticos revelaram que a equação de pseudo segunda-ordem forneceu os melhores ajustes dos dados experimentais, consequentemente, confirmando que o controle do mecanismo de velocidade é a adsorção química (reação química). O mecanismo indicou que a velocidade de adsorção dos metais pelo adsorvente foi dependente da concentração dos íons no adsorvente e a concentração destes adsorvidos no equilíbrio. Os valores das constantes de velocidade determinados para $\mathrm{Cr}$ (VI), Mo (VI) e Se (VI) foram: $1,0 \times 10^{-3}$; $3,0 \times 10^{-2} \mathrm{e} 4,1 \times 10^{-3} \mathrm{~g}(\mathrm{mg} \mathrm{min})^{-1}$, respectivamente. A constante de velocidade de adsorção do Mo (VI) apresentou-se 7 a 30 vezes maior do que a do e $\mathrm{Se}(\mathrm{VI})$ e $\mathrm{Cr}$ (VI).

\section{Isotermas de Adsorção}

Experimentos em batelada foram usados para interpretar o equilíbrio de adsorção. Na presente investigação, os dados foram analisados segundo os modelos de isoterma de Langmuir e Freundlich. O modelo de Langmuir supõe que a superfície do adsorvente possua sítios energéticos idênticos e que cada molécula do adsorbato ocupe um único sítio, conseqüentemente prevê a formação de uma monocamada de cobertura de adsorbato na superficie do adsorvente. Por outro lado, o modelo de Freundlich descreve uma adsorção reversível heterogênea, visto que não restringe a uma monocamada de cobertura do adsorvente ${ }^{[22]}$.

Para o sistema em estudo, a isoterma de Langmuir forneceu o melhor ajuste dos dados experimentais de adsorção. O gráfico mostra a quantidade dos oxiânions metálicos 


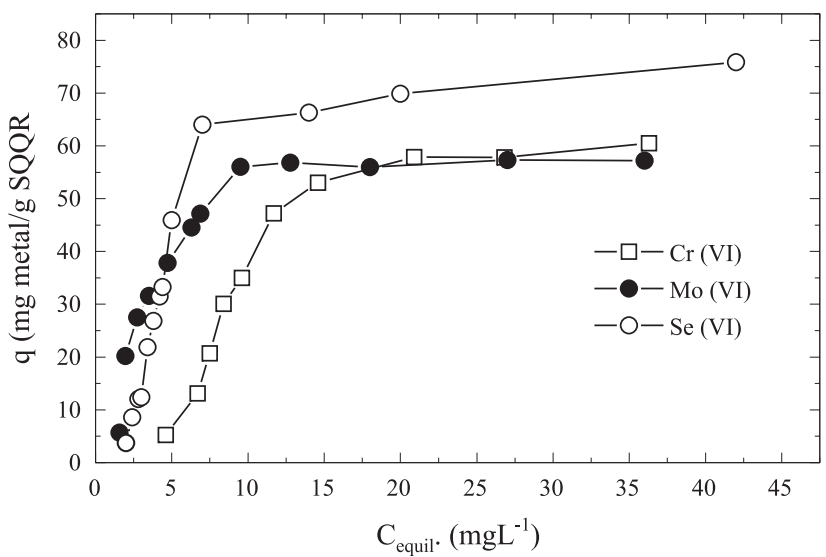

Figura 4. Isotermas de adsorção dos oxiânios cromo, molibdênio e selênio.

$\mathrm{C}_{\mathrm{o}}=10$ a $500 \mathrm{mg} \mathrm{L}^{-1}$; Volume $=50 \mathrm{~mL} ; \mathrm{m}_{\mathrm{SQQR}}=100 \mathrm{mg} ; \mathrm{pH}=4,5$ (Cr e Se) e $\mathrm{pH}=8,0(\mathrm{Mo})$; agitação $=200 \mathrm{rpm}$; temperatura $=25^{\circ} \mathrm{C}$; tempo $=300 \mathrm{~min}$

adsorvidos na superfície do adsorvente e a concentração destes íons na fase aquosa em equilíbrio. Esta relação mostrou que a capacidade de adsorção aumenta com a concentração dos íons na fase aquosa, alcançando progressivamente a saturação em altas concentrações.

A equação de Langmuir foi colocada na sua forma linear mais adequada para a determinação dos parâmetros de adsorção: capacidade máxima de adsorção $\left(\mathrm{q}_{\max }\right.$. $)$ e a constante de Langmuir (K).

$$
\frac{\mathrm{C}_{\mathrm{eq}}}{\mathrm{q}}=\frac{1}{\mathrm{~K} \cdot \mathrm{q}_{\text {max. }}}+\frac{1}{\mathrm{q}_{\text {max. }}}
$$

As curvas lineares obtidas resultaram nas seguintes equações:

$$
\begin{aligned}
& \text { (Cromo): } \mathrm{Y}=0,06508+0,01464 \mathrm{X}(\mathrm{R}=0,99758) \\
& \text { (Molibdênio): } \mathrm{Y}=0,04487+0,01577 \mathrm{X}(\mathrm{R}=0,99633) \\
& \text { (Selênio): } \mathrm{Y}=0,06455+0,01111 \mathrm{X}(\mathrm{R}=0,99973)
\end{aligned}
$$

O coeficiente angular desta equação corresponde a $1 / \mathrm{q}_{\max }$ e o intercepto representa $1 / q_{\max } \mathrm{K}$. A capacidade máxima de saturação da superfície do adsorvente $\left(\mathrm{q}_{\text {max. }}\right)$ revelou que $1 \mathrm{~g}$ de SQQR adsorveu 68,3 mg de Cr (VI) ou 1,30 mmol; 63,4 $\mathrm{mg}$ de Mo (VI) ou 0,66 mmol e 90,0 mg de Se (VI) ou 1,1 mmol. As constantes de Langmuir foram 0,225; 0,351 e 0,172, respectivamente. Pode-se observar pelos valores obtidos em mmol g ${ }^{-1}$ que a capacidade máxima de adsorção dos oxiânions metálicos foi: $\mathrm{Cr}(\mathrm{VI})>\mathrm{Se}(\mathrm{VI})>\mathrm{Mo}(\mathrm{VI})$.

Para comparar a capacidade de adsorção do SQQR foram utilizados vários estudos relatados na literatura. Quian et al. Realizaram estudos de adsorção $\mathrm{Cr}$ (VI) e Se (VI) com quitosana reticulada com epicloridrina. $\mathrm{O} \mathrm{pH}$ ótimo de adsorção foi 3,0 e 4,0 para o $\mathrm{Cr}(\mathrm{VI})$ e Se (VI), respectivamente. As isotermas de adsorção foram ajustadas utilizando o modelo de Langmuir e a capacidade máxima de adsorção foi de 11,3 e $34,5 \mathrm{mg} \mathrm{g}^{-1}$ para o $\mathrm{Cr}(\mathrm{VI})$ e Se (VI), respectivamente. Segundo Babel et al. a quitosana sem reticulação teve uma capacidade de $273 \mathrm{mg} \mathrm{g}^{-1}$ de $\mathrm{Cr}(\mathrm{VI})$, enquanto na forma reticulada a capacidade foi de $78 \mathrm{mg} \mathrm{g}^{-1}$. Guibal obteve na adsorção de $\mathrm{Cr}$ (VI) pela quitosana reticulada uma capacidade de $65 \mathrm{mg}$ por grama. Comparando estes valores com os obtidos neste estudo, a capacidade de adsorção do SQQR foi similar a quitosana reticulada em alguns estudos. A vantagem do uso do SQQR é que este apresenta um grupo catiônico como sítio de adsorção e a quitosana depende do $\mathrm{pH}$ da solução para protonação dos grupos amíno, e assim obter o sítio catiônico na superfície do polímero.

\section{Seletividade e mecanismo de adsorção dos oxiânions pelo SQQR}

A análise semi-quantitativa da habilidade do sal quaternário de quitosana pelos metais foi realizada por energia dispersiva de raio-X (EDX). O espectro de EDX do material sólido contendo os metais adsorvidos (Figura 5) mostrou

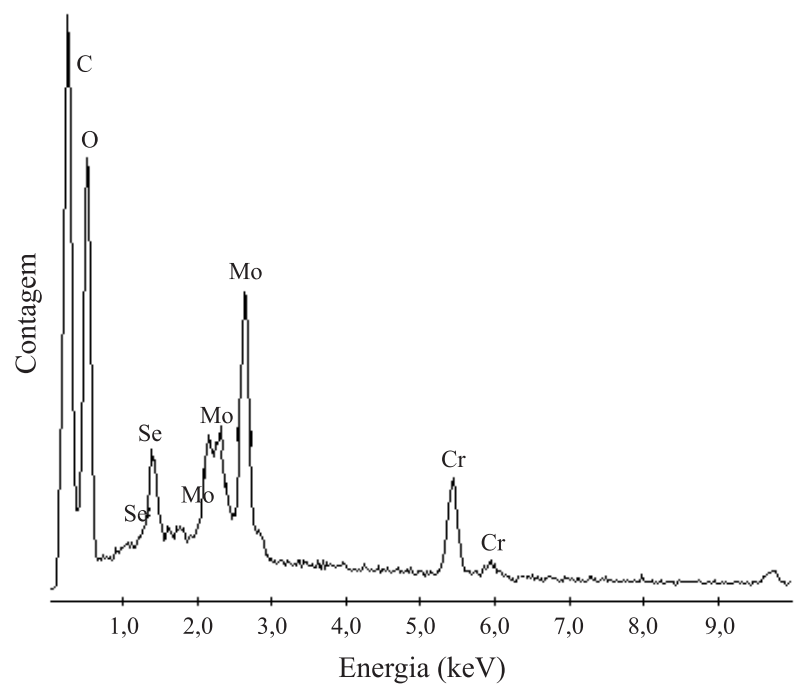

Figura 5. Espectro de EDX para determinação da seletividade dos oxiânios de $\mathrm{Cr}(\mathrm{VI})$, Mo (VI) e Se (VI) pelo SAQQR; $\mathrm{pH}=8,0$

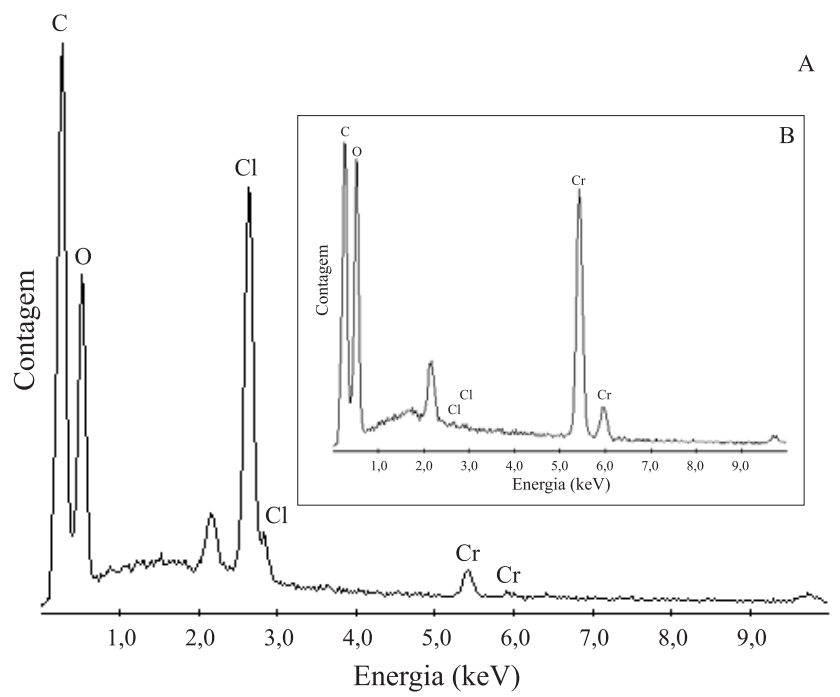

Figura 6. Espectros de EDX de amostras do SQQR utilizando concentrações diferentes de $\mathrm{Cr}$ (VI). A = $25 \mathrm{mg} \mathrm{L}^{-1}$ de $\mathrm{Cr}$ (VI); B $=1000 \mathrm{mg} \mathrm{L}^{-1}$ de $\mathrm{Cr}$ (VI); $\mathrm{pH}=4,5$. 
a seletividade do adsorvente. A partir das porcentagens atômicas a ordem de seletividade foi $\mathrm{Cr}>\mathrm{Mo}>\mathrm{Se}$.

$\mathrm{O}$ mecanismo de adsorção pode ser confirmado pelos espectros de EDX do Cr (VI) adsorvido. Ficou evidenciado, pelas análises semi-quantitativas de cloreto e do oxiânion, o mecanismo de troca iônica entre os dois íons. A Figura 6 ilustra o espectro de EDX do material após o contato com soluções de Cr (VI) 25 e $1000 \mathrm{mg} \mathrm{L}^{-1}$.

\section{Conclusões}

Os estudos de equilíbrio de adsorção mostraram que o sal de amônio quaternário reticulado (SAQQR) foi efetivo como adsorvente para os oxiânions Cr (VI), Mo (VI) e Se (VI) em solução aquosa. Os resultados demonstraram que o processo de adsorção do $\mathrm{Cr}$ (VI) e Se (VI) é dependente do pH e que o mecanismo cinético segue o modelo de pseudo segunda-ordem. Os estudos de EDX revelaram que o principal mecanismo de adsorção é a troca iônica entre os íons $\mathrm{Cl}^{-}$da superfície do polímero pelos oxiânions da solução e a seletividade foi: $\mathrm{Cr}>\mathrm{Mo}>\mathrm{Se}$.

\section{Agradecimentos}

O presente trabalho foi realizado com o apoio do Conselho Nacional de Desenvolvimento Científico e Tecnológico CNPq - Brasil.

\section{Referências Bibliográficas}

1. Costa, C. A - "Remoção de metais pesados por sorção em subproduto do beneficiamento do carvão", Dissertação de Mestrado, Universidade Federal do Rio Grande do Sul, Brasil (2000).

2. Dakiky, M.; Khamis, M.; Manassra, A.; Mereb, M. Advances in Environmental Research, 6, p.533 (2002).

3. Babel S.; Kurniawan, A. T.; - Chemosphere, 54, p. 951 (2004).

4. Jimenez, S. R., Dal Bosco, M. S., Carvalho, A. W. - Química Nova, 27, 5, p.734 (2004).

5. Spinelli,V. A.; Fávere, V. T.; Laranjeira, M. C. M. - Reactive $\&$ functional polymers, 61, 3, p.347 (2004).

6. Guibal, E. - Separation and Purification Technology, 38, p.43 (2004).
7. Liu, X. D.; Tokura, S.; Haruki, M.; Nishi, N.; Sakari, N. Carbohydrate Polymer, 49, p.103 (2002)

8. Rhazi, M..; Desbrières; Tolaimate, A.; Rinaudo, M.; Vottero, P.; Alagui, A.; El Meray, M. - European Polymer journal, 38, p.1523 (2002).

9. Blais, J. F.; Shen, S.; Meunier, N.; Tyagi, R. D. Enironmental Technology, 24, p.205 (2003).

10. Qian, S.; Huang, G.; Jiang, J.; He, F.; Wang, Y. - Journal of Applied Polymer Science, 77, 3215 (2000).

11. Chassary, P.; Vicent, T.; Guibal, E. - Reactive \& Functional Polymers, 60, p.137 (2004).

12. Lang, G. R., Wenbel, H., Konrad, E. Quaternization of chitosan with glycidyl trimethyl ammonium chloride. Patent number: 4.822.598, Apr. 18, 1989, United States Patent.

13. Kin, H. C., Choi, W. J., Chun, J. H., Choi, S. K. International Journal of Biological Macromolecules, 22, p .387 (1996).

14. Quin, C. Xiao, Q., Li, H., Fang, M., Liu, Y., Chen, X., Li, Q. - International Journal of Biological Macromolecules, 34, p. 121 (2004).

15. Xu, Y., Du, Y., Huang, R., Gao, L. - Biomaterials, 24, p.5015 (2003).

16. Domard, A., Gey, C., Rinaud, M., Terrasin. - International Journal of Biological Macromolecules, 9, p.233 (1987).

17. Lim, H. S., Hudson, M. S. - Carbohydrate Research, 339 , p.313 (2004).

18. Burriel, M. F.; Conde, L. F.; Jimeno, A. S.; Méndez, H. J. - "Quimica Analitica Cualitativa", Editorial Paraninfo S. A., Móstoles, Madrid (1994).

19. Wu, C.F., Tseng, L.R., Juang, S.R. - Water Research, 35, 3, p.613 (2001).

20. Ho, S. Y.; Mckay, G. - Water Research, 34,3, p.735 (2000).

21. Sag, Y.; Aktay, Y. - Biochemical Engineering Journal, 12, p.143 (2002).

22. Mckay, G. - "Use of adsorbents for the removal of pollutants from wastewaters", CRC Press, Inc., New York (1996).

Enviado: 08/11/04 Reenviado: 08/04/05 Aprovado: 09/05/05 